\title{
Surgical Rib Fixation: Five-Year Experience from an Australian Trauma Centre
}

\author{
Bhavik Patel*, Gary Hung, Andrie Stroebel and Martin Wullschleger \\ Trauma Service, Gold Coast University Hospital, Queensland, Australia
}

${ }^{\star}$ Corresponding author: Bhavik Patel, MPhil (Research), FRACS, Trauma Service, Gold Coast University Hospital, Queensland 4125, Australia; Email: drbhavikpatel@ hotmail.com

Received: September 06, 2019; Accepted: September 19, 2019; Published: September 22, 2019;

\begin{abstract}
Background

Literature suggests surgical rib fixation in the acute phase of injury leads to positive outcomes. We have implemented this practice since 2014 and detail the outcomes of rib fixation from our institution.

\section{Methods}

We implement a multidisciplinary team management for chest injury. Failure to progress despite maximum intervention by the pain specialist is identified early leading to operative intervention. Retrospective 5-year review was performed on patients undergoing surgical rib fixation looking into the effect on pain scores, length of in hospital stay, post-operative complications and follow up.
\end{abstract}

\section{Results}

Thirty-seven cases (81\% males) with a mean age of 56 met the inclusion criteria. All patients underwent rib fixation within 96 hours of admission. Of all included patients, $57 \%(\mathrm{n}=21)$ required intensive care unit admission for ventilatory support. Of this subset of patients, $66 \%(\mathrm{n}=14)$ were discharged to ward management within 48 hours, the remaining seven patients required ongoing support secondary to co-existing injuries requiring ongoing management in the unit. Postoperatively, pain scores reduced at median of 2 days with a standard deviation of 2.5 days. The in-hospital stay also reduced by 155 hours ( 6 days) over the period of 5 years. There were two early complications in the series, but no mortality. At 12 months post operatively, both clinical and radiological follow up suggested no hardware or residual pulmonary complications.

\section{Conclusion}

This single institution study presents the early results of surgical rib fixation with satisfactory outcomes and minimal complications. Ongoing follow-up will provide a more detailed analysis of long-term outcomes

\section{Background}

Rib fractures are present in about $21 \%$ of blunt chest trauma patients presenting to Australian hospitals [1]. The number of ribs fractured and the complexity of fracture pattern are associated with an increase in morbidity and mortality [2,3]. Traditionally rib fractures are managed with focus on adequate pain control, oxygen supplementation and early chest physiotherapy [4]. The management of complex rib fractures by traditional means can be challenging as these patients might require respiratory support in the intensive care unit with prolonged hospital stays during the recovery phase [5].

Open Surgical Rib Fixation (SRF) is being used to manage complex rib fractures and follows the same principle of orthopaedic reduction and fixation of fractured bones to reduce pain by return of structural stability of the chest wall which also improves ventilatory efforts leading to reduced pulmonary complications and prevent delayed union [6-8]. Interventions can be performed in conjunction with SRF such as placement of analgesic catheters, evacuation of retained haemothorax and exploration of other thoracic pathologies such as lung lacerations and integrity of the diaphragm ${ }^{2}$. The benefits of SRF for complex rib fractures has been recognised in reducing pain and improving physical function $[6,7]$.

The Gold Coast University Hospital (GCUH) is a verified Level I Trauma Centre by our Royal Australasian College of Surgeons (RACS). Over the last 5 years, 10,545 patients were managed by the service out of which $2070(20 \%)$ presented with blunt chest wall injury. Or institution promotes multidisciplinary team approach in the management of these patients. Pain specialist team would monitor subjective pain scores for the duration of stay recorded utilising numeric/verbal rating scale [10]. The days required for the patient to have subjective pain improvement by a drop in the pain brackets of severe (10-7), moderate (6-4), mild (1-3) or none (0). Following exhaustion of all modalities of pain relief within the first 96 hours of admission to hospital, patients would be recruited to the surgical pathway. 


\section{Surgical Technique}

In our institution, a standardized SRF technique had been developed by surgeon experience and with adaption to literature. Patients for consideration of SRF have three-dimensional computed tomography reconstruction of the chest wall for operative planning [9]. Anaesthesia is administered via single lumen tube with the patient in lateral position on bean mattress. A minimal muscle splitting surgical approach is performed using a wound protector (ALEXIS - Applied Medical, CA, USA) with minimal rib dissection and preservation of the periosteum [6], followed by reduction and plating (MatrixRib ${ }^{\bullet}$ Fixation System, West Chester, PA, USA or Rib Loc ${ }^{\circledR}$ Rib Fracture Plating System, Acute Innovations, Hillsboro, OR, USA) of the fractures depending on site of fractures. The pleural cavity is then lavaged with saline and a curved intercostal catheter inserted. Post-operative chest X-ray determines the need for suction on the drainage system. Paravertebral blocks are either left in situ or replaced at the end of the procedure.

The aim of this study is to describe the outcomes of surgical rib fixations looking at in hospital length of stay, pain scores and duration of intensive care stay over a period of five years at our institution.

\section{Methods}

\section{Design and Setting}

Following local ethics committee approval (LNR/2018/ QGC/49835), a retrospective chart review was conducted on all SRF cases performed from July 2014 to December 2018 at Gold Coast University Hospital.

\section{Participants}

We included all patients who underwent surgical rib fixations irrespective of their other injury status. Penetrating chest wall Injuries leading to rib fractures and blunt injury induced fractures managed without surgical intervention were not included in this study.

\section{Outcomes}

Primary outcome was to evaluate length of stay in hospital and secondary outcome being improvement in pain scores, reduction in ventilatory days and complications.

\section{Data Collection}

Data was retrieved via the prospectively collected trauma registry managed by the trauma service at GCUH.

\section{Data Analysis}

Statistical analysis was performed using IBM SPSS statistics for windows. Statistical analysis was performed using t-test (two-sample assuming unequal variances), specifically to evaluate the difference in outcomes in pain, length of stay in hospital and ventilatory days. Descriptive analysis was performed on non-comparative values.

\section{Results}

Thirty-seven cases ( $81 \%$ males) with a mean age of 56 met the inclusion criteria. Flow Chart 1 displays details of recruitment.
The chest Abbreviated Injury Scale (AIS) scores were $\geq 3$ for all cases. The mean Injury Severity Score (ISS) was 21. Figure 1 displays distribution of ISS/New Injury Severity Score (NISS) over the period of 5 years.

All patients underwent surgical rib fixation within 96 hours of admission. The number of operative cases progressed from three annually in 2014/2015 to twenty in 2018. In 2014, at the beginning of the study, pain not controlled following maximum intervention from the pain specialist was the predominant indication for intervention however as the duration of the study progressed surgical interventions were also performed for chest wall instability. Five patients were excluded from the study as for more than 48 hours either pre- or post-procedure secondary to their injuries it would not be possible to document their pain scores.

Of all included patients, $57 \%(\mathrm{n}=21)$ were admitted pre-as well as post-operative to intensive care unit admission for respiratory support. Of this subset of patients, $66 \%(n=14)$ were discharged to ward within 48 hours. The remaining seven patients required ongoing support in the unit for management of their co-existing injuries. The median days after SRF where patients described pain score improvement were 2 with a standard deviation of 2.5 days.

The length of in hospital stay over a period of 5 years reduced from 546 to 391 hours, a difference of 155 hours (6 days). The overall median length of in hospital stay was 292 hours with a Standard deviation of 313 hours. Patients sustaining concurrent severe head injury if excluded, the length of stay reduced from 401 to 261 hours difference of 140 hours (5 days).

As time progressed, we undertook surgical fixation of the more anatomically challenging posterior $(10 \%)$ and bilateral (8\%) rib fractures.

Over the course of 5 years, follow-up rate at 12 months progressively increased from $75 \%$ to $85 \%$ with no hardware or pulmonary complications reported on plain chest $\mathrm{x}$-rays.

Complications in this case series consisted of a postoperative bleed in a posterior rib fixation patient requiring operative intervention with second intercostal catheter placement and a superficial wound infection managed non-operatively with intravenous antibiotics. Non-procedure complications included pneumonia (2), pulmonary embolism (1) and mortality due to traumatic brain injury (1). Thus, overall complication rate was $16.2 \%$ with an overall procedure related complication rate of $5.4 \%$.

\section{Discussion}

Since the introduction of surgical rib fixation at our institution, there has been a steady increase in the number of cases performed annually. This could not only be attributed to the increasing confidence of the surgeon performing the procedure but also to patient outcomes in terms of reduction in pain scores, reduced ventilatory days and decreased length of in hospital stay. 


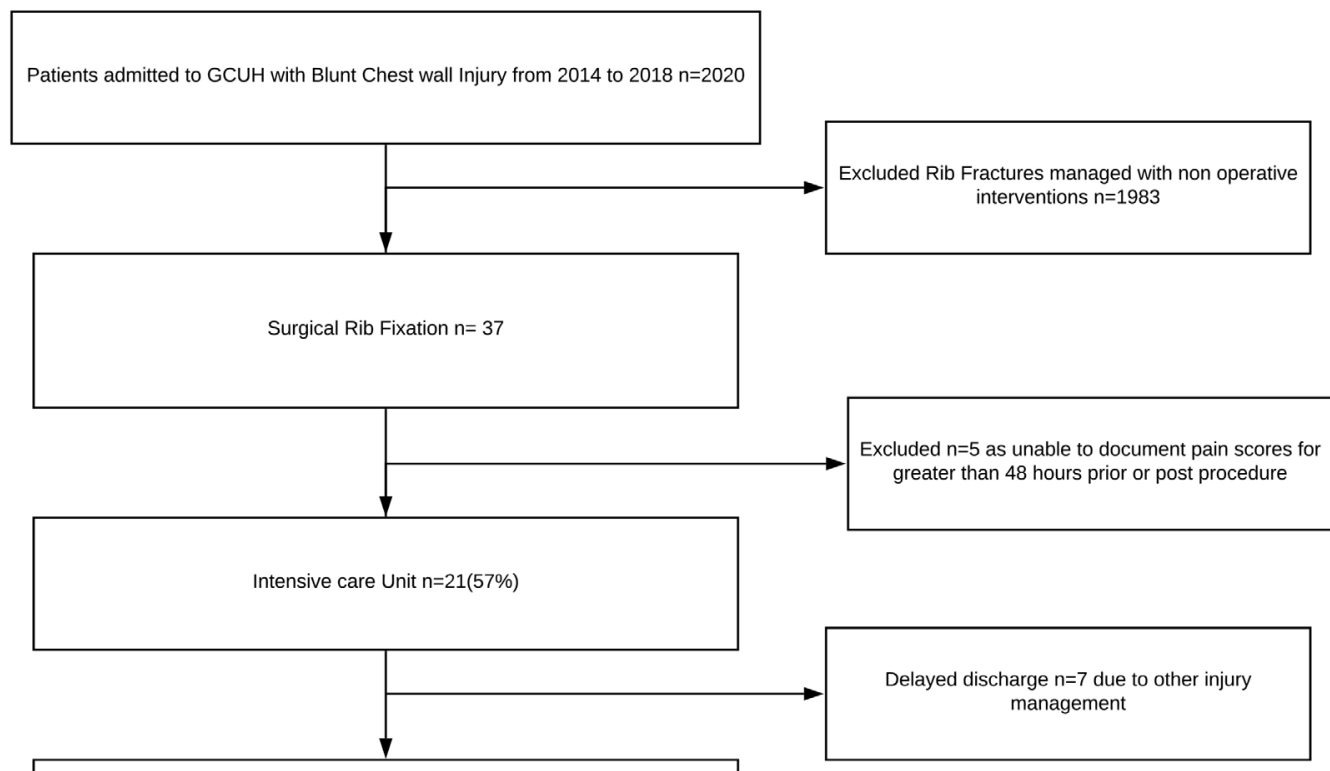

Discharge to ward within 48 hours $n=14(66 \%)$

ISS and NISS Distribution 2013/2018 Rib Fixation population

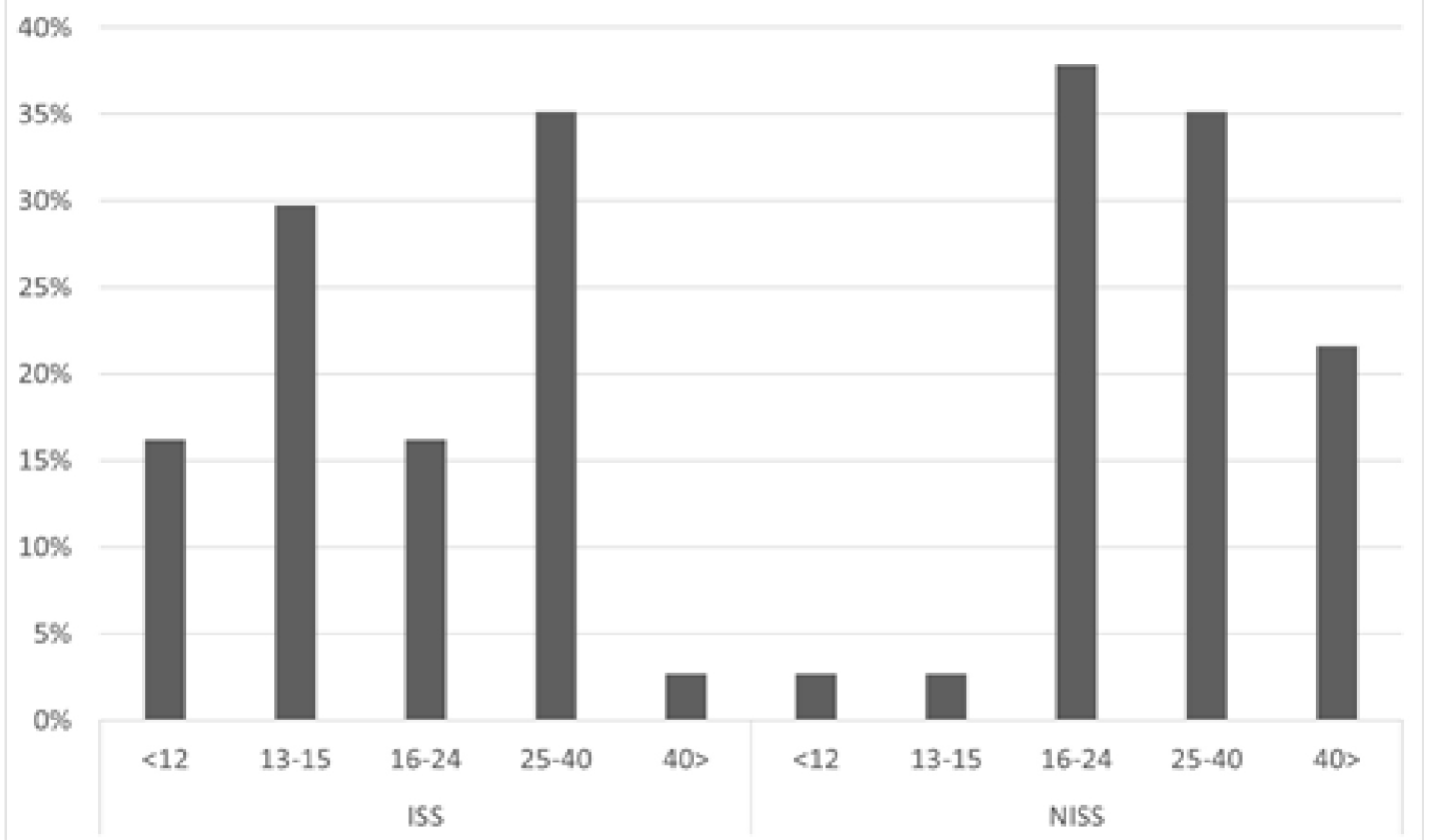

Figure 1. Distribution of ISS and NISS over a 5- Year period 


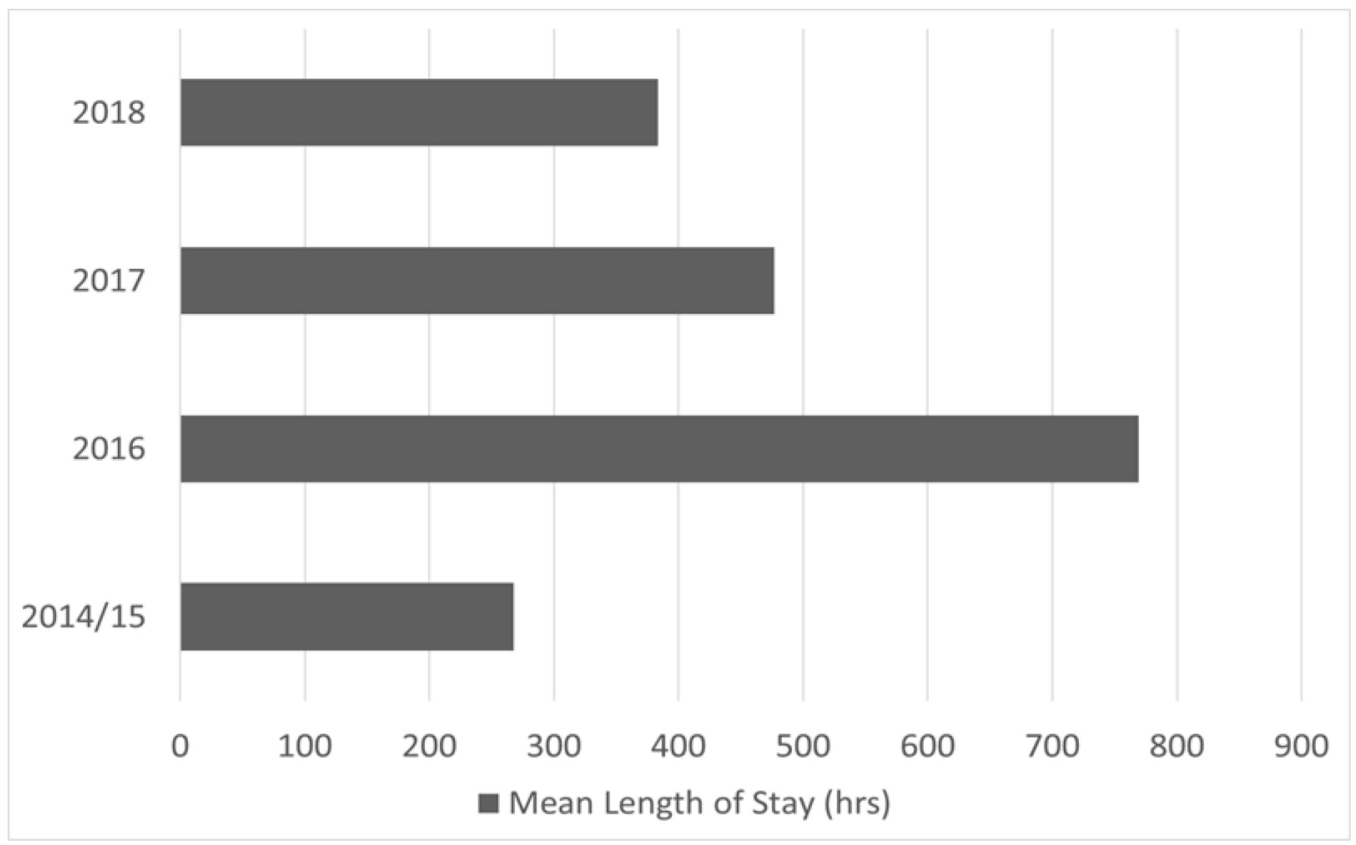

Figure 2. Demonstrates the length of in-hospital stay.

Pain is the main complaint of rib fracture patients and management of this is a challenge often with complex fracture patterns. Once all supportive pain management options are exhausted, surgical rib fixation is considered.

The average cost of patients acutely admitted to public hospitals in Queensland, Australia is about $\$ 8561$ for 4 days [11]. The postoperative time to improvement in subjective pain scores was 2 days, which might have a direct link in reduction of the length of in hospital stay by 155 hours ( 6 days). This study suggests direct link to subjective pain relief following surgery and reduction in length of hospital stay leading to reduction in costs of hospital stay [14].

The post-operative time to improvement in subjective pain experienced has decreased over the period of this study. This may be due to refinement in surgical technique by reducing the size of surgical incision and minimum muscle dissection as more cases were performed. Another confounding factor for subjective pain score improvement could be the increased experience of the pain specialist and allied health professionals in managing these subsets of patients.

Literature review suggests acute recovery from complex rib fractures can be measured by length of hospital stay [13]. In the identified patients who underwent SRF, the mean HLOS has shown a steady decrease by 155 hours (6 days). When patients with concurrent severe head injury is excluded from the groups, the mean difference still favours the surgical group by 140 hours (5 days) suggesting similarity in local as well as international results $[13,14]$.

It is recommended for novice surgeons to perform the technique on antero-lateral rib fractures [5]. As the experience in surgical technique was gained, confidence in performing the more challenging posterior and bilateral rib fractures also increased.
Some clinical studies are substantially smaller and often lack sufficient statistical power to detect clinically meaningful differences in operative mortality rates. Moreover, there is little evidence from these clinical studies to suggest there are important volume-related differences in the case mix (i.e. those low-volume providers. Although we cannot rule out confounders by unmeasured characteristics of the patients in our study, there is no reason to believe that such confounders would affect our analyses of hospital and surgeon volume [12].

\section{Limitations}

There are certain limitations of this study with the most distinct being the retrospective nature of this study which includes small cohort of patients at a single institution. Major chest trauma is usually associated with other injuries and the severity of this may be a confounder to the true in hospital length of stay. A multi institutional prospective study might provide us with suggestions into the outcomes of this surgical procedure.

\section{Conclusion}

Preliminary data analysis from our institution suggests, we have increased our incidence for this procedure with reduced in hospital length of stay and minimal impact to complications. We hope to see consistent results as case numbers increase in the following years and for fellow institutions to support these outcomes.

\section{Declaration}

Ethical statement: Study was carried out only after approval from Local Ethics Committee (LNR/2018/QGC/49835)

Consent for publication: Retrospective de identified chart analysis only so no consent from patients 
Availability of data and material: On request de identified data can be accessed

Competing interests: Bhavik Patel, Gary Hung, Andrie Stroebel and Martin Wullschleger have no competing interest

This study has been presented as a poster at The Annual Summit of Chest Wall Injury Society, Santa Fe, New Mexico, USA- 2019.

\section{Authors' Contributions}

Bhavik Patel- Devise Idea, Draft Manuscript, Analysis of data, Submission and Corresponding Author

\section{Gary Hung- Collect data, Analysis of data, Draft Manuscript}

Andrie Stroebel- Initial Phase Cardiothoracic Surgeon for Second Opinion on operative cases

Martin Wullschleger - Initial Phase Surgeon and Correction of Manuscript

\section{References}

1. Cameron P, Dziukas L, Hadj A, Clark P, Hooper S (1996) Rib fractures in major trauma. Aust N Z J Surg 66: 530-534.

2. Witt CE, Bulger EM (2017) Comprehensive approach to the management of the patient with multiple rib fractures: a review and introduction of a bundled rib fracture management protocol. Trauma Surgery \& Acute Care Open 2: 1-7.

3. Chien CY, Chen YH, Han ST, et al. The number of displaced rib fractures is more predictive for complications in chest trauma patients. Scand J Trauma Resusc Emerg Med 25: 19.

4. Kane ED, Jeremitsky E, Pieracci FM, Majercik S, Doben AR (2017) Quantifying and exploring the recent national increase in surgical stabilization of rib fractures. $J$ Trauma Acute Care Surg. 83: 1047-52.

5. de Campos JRM, White T (2018) Chest wall stablization in trauma patients: when, when, and how? J Thorac Dis 10: S951-S962

6. Pieracci FM, Majercik S, Ali-Osman F, et al. (2017) Consensus statement: Surgical stabilization of rib fractures rib fracture colloquium clinical practice guidelines. Injury 48: 307-21

7. Fagevik Olsén M, Slobo M, Klarin L, et al. (2016) Physical function and pain after surgical or conservative management of multiple rib fractures - a follow-up study. Scand J Trauma Resusc Emerg Med 24: 128.

8. Kaplan DJ, Begly J, Tejwani N. Multipe (2017) Rib Nonunion: Open Reduction and Internal Fixation and Iliac Crest Bone Graft Aspirate. J Orthop Trauma 3: S34-5.

9. Benjamin R. Pulley, Benjamin C. Taylor, Terry Ty Fowler, Neysa Dominguez, Thai Q (2017) Trinh. Utility of three-dimensional computed tomography for the surgical management of rib fractures. J Trauma Acute Care Surg 78: 530-533.

10. Breivik et al (2008) Assessment of Pain. Br J Anaesth 101: 17-24.

11. Independent Hospital Pricing Authority. National Hospital Cost Data Collection. Australian Public Hospitals Cost Report 2013-2014.

12. Birkmeyer JD, Stukel TA, Siewers AE, Goodney PP, Wennberg DE, Lucas FL (2003) Surgeon Volume and Operative Mortality in the United States. $N$ Engl $J$ Med 349: 2117-27

13. Silvana F. Marasco, Andrew R. Davies, Jamie Cooper et al. (2013) Prospective Randomized Controlled Trial of Operative Rib Fixation in Traumatic Flail Chest. Journal of the American College of Surgeons 216: 924-932.

14. Sarah Majercik, Emily Wilson, Scott Gardner, Steven Granger, Don H. VanBoerum, et al. (2015) In-hospital outcomes and costs of surgical stabilization versus nonoperative management of severe rib fractures. J Trauma Acute Care Surg 79: $537-539$.

\section{Citation:}

Patel B, Hung G, Stroebel A, Wullschleger M (2019) Surgical Rib Fixation: Five-Year Experience from an Australian Trauma Centre. Integr J Orthop Traumatol Volume 2 (5): 1-5. 